\title{
Uses of Ultrasound and Doppler in Assessment of Endometrial Receptivity in Patients of Unexplained Infertility
}

Emad Abd El-Rahman El-Tamamy, Mofeed Fawzy Mohamed and Badawy Ezzat Hamza*

Obstetrics and Gynecology Department, Faculty of Medicine, Al Azhar University

*Corresponding Author: Badawy Ezzat Hamza,Phone No.: (+2) 01100399809, email: bodyatef07.ba@gmail.com

\begin{abstract}
Background: although the majority of infertile couples was found to have an identifiable male or female factor, $15-30 \%$ was categorized as having "unexplained infertility". The endometrium is considered to be a key determinant in successful implantation. Endometrial receptivity is regulated by many factors including endometrial perfusion. Aim of the Work: this study aimed to detect the role of the two dimensional ultrasound and Doppler ultrasonographic uterine markers of implantation in patients of unexplained infertility compared to fertile participants. Patients and Methods: the study was performed on 50 patients presented to the outpatient clinic in Al-Hussein, Bab El-Shearia (Sayed Galal) Hospitals, Al-Azhar University from October 2017 to August 2018 and they were categorized into two groups:

- Group A (Patients group): it was included 30 participants presented with unexplained infertility.

- Group B) (Control group) who included 20 fertile women coming for contraception.

- Results: there was a significant statistical difference as regard the endometrial thickness between cases and controls, and the controls had a thicker endometrium. Lower pulsatility index (PI) in uterine artery in the controls compared to the infertile group. Increased sub endometrial vascularization in the controls compared to the infertile group. Conclusion: ultrasound as well as Doppler studies of the endometrial and subendometrial vascularization had a role in predicting endometrial receptivity. Uterine artery Doppler study and the endometrial thickness measurement had a role in detection of uterine receptivity.
\end{abstract}

Keywords: endometrial receptivity, unexplained infertility, ultrasound, doppler

\section{INTRODUCTION}

Ultrasound examination of the endometrium is a commonly used non-invasive method of assessing endometrial receptivity during infertility treatment. Assessment of the endometrium, however, is usually limited to measurement of its thickness and description of its appearance, and these parameters may not be specific indicators of implantation ${ }^{(1)}$.

Doppler ultrasound assessment of uterine blood flow appears to be more informative, but Doppler study of uterine arteries does not reflect the actual blood flow to the endometrium. Endometrial and sub endometrial blood flows can be more objectively and reliably measured with threedimensional (3D) power Doppler ultrasound (2).Ultrasound imaging technology brings a new insight to the cyclic changes in the endometrium and offers the potential to assess the probability of embryo transfer in natural and artificial reproductive cycle, however the data are diverse and frequently conflict in their analysis and conclusions. Ultrasound technology provides a rapid, noninvasive endometrial evaluation (3). Current practice depends on two dimensional trans-vaginal ultrasound for monitoring ovulation.Three dimensional ultrasound is a revolution in the non-invasive monitoring of follicular development. Owing to the presence of the third dimension (coronal plane), three dimensional ultrasound can perform a precise measurement of the area and volume of each follicle and the endometrium ${ }^{(4)}$. There is a reasonable certainty that women with heterogenous endometrial lining of less than 6 $\mathrm{mm}$ will rarely conceive and lack of endometrial contraction plays a role in the establishment of pregnancy, so these ultrasonographic imaging techniques are used as predictors of uterine receptivity. Also different utero-ovarian blood flow changes during the peri-implantation period occur in conception and non-conception cycles ${ }^{(5)}$.Doppler assessment of uterine arterial resistance can help to determine a time interval within the menstrual cycle that is of optimal endometrial status for embryo transfer in assisted conception programs. Delay in achieving adequate uterine perfusion during the temporal window of implantation may have an impact on endometrial receptivity ${ }^{(6)}$.Presence of both endometrial and sub-endometrial flow is indicative of good endometrial receptivity, whereas the absence of both represents a poor 
uterine environment which is very common in old age, those with thinner endometrium and those who had higher uterine arterial resistance compared with those with detectable flow ${ }^{(7)}$. Endometrium is better represented as volume with high positive and negative predictive value of endometrial receptivity. The high degree of endometrial perfusion shown by the colour Doppler ultrasound and the three dimensional power Doppler ultrasound on the day of embryo transfer can indicate favorable endometrial milieu for pregnancy ${ }^{(8)}$.Endometrial receptivity is important for optimizing the results of assisted reproductive technology (ART), it is critical to decide the timing for embryo transfer (ET) that would best correspond with the implantation window. Biochemical evaluation of endometrial function at the time of implantation has been tried in recent years, but no conclusion about its clinical significance in the assessment of endometrial receptivity after ART can be drawn at the present time Furthermore, most cases involved invasive procedures which are not suitable in the treatment cycles ${ }^{(9)}$. With the introduction of high-resolution transvaginal probes, the noninvasiveness and accessibility of sonography have made it particularly suitable for serial follow-up throughout the menstrual cycle. Assessment of uteroovarian blood flow is a potentially valuable method for evaluating the functional capacity of the endometrium during the window of implantation ${ }^{(10)}$.Implantation remains one of the last frontiers of reproductive medicine and there is currently a huge amount of research in this area. It's clearly a complex process and the scientific understanding of normal implantation lags far behind what is known in areas of reproductive function as fertilization ${ }^{(11)}$. The growth of our knowledge about this process will allow authors to improve implantation rates in infertile patients or in contrast to develop novel anti-implantation or interception technologies. Implantation is the most important limiting factor in human reproduction. Only one-fourth of all fertilized ova will generate a live human being and half of failures occur at the time of implantation ${ }^{(12)}$.It seems that synchronized development of a healthy blastocyst and receptive endometrium is vital for the implantation process. The implantation window which is highly dependent on a receptive endometrium, was suggested to be on day 20 to day 24 of 28 days cycle or between day $\mathrm{LH}+7$ \& day $\mathrm{LH}+11$ in assisted conception cycle ${ }^{{ }^{(13)} \text {.Ultrasound }}$ imaging technology brings a new insight to the cyclic changes in the endometrium and offers the potential to assess the probability of embryo transfer in natural and artificial reproductive cycle, however the data are diverse and frequently conflict in their analysis and conclusions. Ultrasound technology provides a rapid, noninvasive endometrial evaluation ${ }^{(3)}$.It is interesting to note that impressive advances are being made in our understanding of the timing of protein expression in the endometrial glands and stroma. Potential markers for uterine receptivity include osteopontin, cytokines (leukemia inhibitory factor, colony-stimulating factor-1, and interleukin-1), cell adhesion molecules (the integrins), and the L-selectin ligand, which has been proposed to mediate embryo attachment. In the future, endometrial biopsies may again become part of the diagnostic evaluation if expression patterns of these proteins prove to be predictive of endometrial receptivity ${ }^{(\mathbf{1 4})}$.

\section{AIM of the WORK}

The aim of the work was to detect the role of the two dimensional ultrasound and Doppler ultrasonographic uterine markers of implantation in patients of unexplained infertility compared to fertile participants.

\section{PATIENTS and METHODS}

The study was performed on 50 patients presented to the outpatient clinic in AlHussein, Bab El-Shearia (Sayed Galal) Hospitals, Al-Azhar University from October 2017 to August 2018 and was divided into two groups:

- Group A: (Patients group): included 30 participants presented with unexplained infertility.

- Group B: (Control group) included 20 fertile women coming for contraception.

At the time of initial recruitment, the purpose of the study was adequately explained to each participant. All procedures followed were in accordance with ethical standard of responsible committee, informed and written consent were taken from all patients.

\section{- Type of study:}

Prospective cohort study

\section{Inclusion criteria:}

- Age group: 20 - 35 years old.

- Complaining of primary infertility for more than one year or with history of chemical pregnancy without established clinical pregnancy. 
- Male factor was excluded by recent semen analysis (within the last year) and according to WHO strict criteria 2010.

- Coital causes of infertility were excluded through sexual history.

- Peritoneal factor of infertility was excluded by the absence of history of previous abdominal or pelvic operations and by recent diagnostic laparoscopy that show no organic lesion, no pelvic or peritubal adhesions.

\section{Exclusion Criteria:}

The patients with any of the following criteria were excluded from the study:

- Patients younger than 20 years or older than 35 years.

- There was a coital cause of infertility.

- Patients who had a history of previous abdominal or pelvic operations or cesarean section.

- Patients with history of endometriosis.

- Laboratory evidence of antiphospholipid syndrome or thrombophillias.

All of them were subjected to the following:

1- Two dimensional ultrasound

2- Doppler and power Doppler studies

Type of ultrasound machine:

All ultrasound scans two dimensional (2D) and three dimensional (3D) was done using transvaginal probe transducer. First, the uterus was visualized in the $\mathrm{B}$ mode.

All data of the uterus and ovaries were obtained. The system was then switched to the volume mode.

\section{Technique of trans-vaginal ultrasound:}

- The examination table:

For the scanning procedure an in-line transducer probe (a probe that had an end firing scan head with its shaft and handle on the same axis), so a flat ultrasound examination table would be appropriate.The elevated thighs enabled free movement of the probe in the horizontal plane by the operator. Trendelenberg position (pelvis elevated and upper body too low) was avoided since the minimal amount of pelvic fluid that was often present in the normal examination can help in outlining pelvic organs. For this reason, the use of slightly reversed trendelenberg position during examination was preferred.

\section{- Preparation of the patient for ultrasound examination:}

The transvaginal examination was conducted with the same respect for privacy afforded to the patient during pelvic examination performed in a gynecology clinic. Because the transvaginal probe was placed close to the uterus, with particularly no intervening bowel, the presence of a full bladder is not necessary before the beginning of the scan.

\section{The equipment and the transducer:}

The vaginal probe was covered with an examining glove containing a small amount of gel. The gel ensured good contact between the transducer and the overlying glove. Care was taken to avoid trapping of any air bubbles, which might have created unwanted artifacts on the screen. Cross infection was prevented by the use of probe cover and disinfectants. The Probe with straight handles provided a better transverse view of the lateral portions of the adnexae. This probe (with frequency of 5-7.5 $\mathrm{MHz}$ ) limited the field of view to within 10-12 $\mathrm{cm}$. depth from the probe.

All the patients were subjected to the following in the late follicular phase:

- Two dimensional ultrasound were performed to evaluate: The endometrial thickness.

- Ultrasound scan when the mean diameter of lead follicle was $>14 \mathrm{~mm}$, to measure its mean diameter.

- The uterine artery Doppler study was done with calculation of the Doppler marker: pulsatility index (PI).

- Power Doppler was done to assess the presence or absence of subendometrial blood flow.

The statistical methods:

Data were statistically described in terms of range, mean \pm standard deviation $( \pm$ $\mathrm{SD})$, median, frequencies (number of cases) and percentages when appropriate. Comparison of quantitative variables between the study groups was done using Student $t$ test for independent samples. For comparing categorical data, Chi square $\left(\chi^{2}\right)$ test was performed. Exact test was used instead when the expected frequency is less than 5. A probability value ( $p$ value) less than 0.05 was considered statistically significant. All statistical calculations were done using computer programs Microsoft Excel 2003 (Microsoft Corporation, NY, USA) and SPSS (Statistical Package for the Social Science; SPSS Inc., Chicago, IL, USA) version 15 for Microsoft Windows.

\section{RESULTS}


This study was carried out on 50 participants who were categorized into two groups:

Group A: (The studied group with 30 participants), 26 with unexplained primary infertility for more than 1 year and 4 with history of chemical pregnancy without established clinical pregnancy. The duration of infertility ranged from 1-15 years with a mean of $5.95 \pm 3.83$ years.

Group B: (Control group with 20 participants), they were fertile women coming for contraception. The age of group A ranged from 21-35 years with a mean of $26.70 \pm 4.22$ years, while the age of group B ranged from 2335 years with a mean of $29.78 \pm 3.43$ years. So, there was a statistically insignificant difference between the studied and control groups as regard the age ( $\rho: 0.05)$ Table (1).

Table 1: age distribution of the studied and control groups

\begin{tabular}{|l|l|l|}
\hline & $\begin{array}{l}\text { Age of Study } \\
\text { Group }(\mathbf{N = 3 0})\end{array}$ & $\begin{array}{l}\text { Age of Control } \\
\text { Group }(\mathbf{N = 2 0})\end{array}$ \\
\hline Maximum & 35 & 35 \\
\hline Minimum & 21 & 23 \\
\hline Mean & 26.70 & 29.78 \\
\hline SD & 4.22 & 3.43 \\
\hline Analysis & $P: 0.05$ & \\
\hline
\end{tabular}

All participants were subjected to the following at Al-Hussein Hospital in the late follicular phase:

- Two dimensional ultrasound was performed to evaluate the endometrial thickness.

- Ultrasound scan when the mean diameter of lead follicle was $>14 \mathrm{~mm}$ to measure its mean diameter.

- The uterine artery Doppler study was done with calculation of the Doppler marker: pulsatility index (PI).

- Power Doppler was done to assess the presence or absence of subendometrial blood flow.

According to the endometrial thickness the Group (A) had a minimum $=3 \mathrm{~mm}$, maximum $=12.5 \mathrm{~mm}$ and mean $=6.2 \pm 2.39 \mathrm{~mm}$, while in Group (B) the minimum $=5 \mathrm{~mm}$, maximum $=16 \mathrm{~mm}$ and mean $=8.6 \pm 3.98 \mathrm{~mm}$

Table (2).

Table 2: relation of the endometrial thickness in both the studied and control groups in the late follicular phase

\begin{tabular}{|l|l|l|}
\hline & $\begin{array}{l}\text { Endometrial } \\
\text { Thickness in the } \\
\text { Study } \\
\text { group }(\mathbf{N}=\mathbf{3 0})\end{array}$ & $\begin{array}{l}\text { Endometrial } \\
\text { Thickness in the } \\
\text { Control } \\
\text { group }(\mathbf{N}=\mathbf{2 0})\end{array}$ \\
\hline Maximum & 12.5 & 16 \\
\hline
\end{tabular}

\begin{tabular}{|l|l|l|}
\hline Minimum & 3.00 & 5 \\
\hline Mean & 6.2 & 8.6 \\
\hline SD & 2.39 & 3.98 \\
\hline Analysis & P: 0.01 \\
\hline \multicolumn{2}{|c|}{ The difference was statistically }
\end{tabular}
significant between the studied and control groups as regard of the endometrial thickness in the late follicular phase (P 0.01).

Concerning uterine artery Doppler (PI) group $\mathrm{A}$ had a minimum $=0.84$, maximum $=4.70$ and mean $=2.42 \pm 0.96$, while in group $B$ the minimum $=1.17$, maximum $=2.70$ and mean $=1.88 \pm 0.44$ (Table 3).

Table 3: relation of one of the uterine artery Doppler markers: pulsatility index (PI) in both the studied and control groups the late follicular phase

\begin{tabular}{|l|l|l|}
\hline & $\begin{array}{l}\text { (PI) in Study } \\
\text { group }(\mathbf{N}=\mathbf{3 0})\end{array}$ & $\begin{array}{l}(\mathbf{P I} \text { in Control } \\
\text { group }(\mathbf{N = 2 0})\end{array}$ \\
\hline Maximum & 4.70 & 2.70 \\
\hline Minimum & 0.84 & 1.17 \\
\hline Mean & 2.42 & 1.88 \\
\hline SD & 0.96 & 0.44 \\
\hline Analysis & P: 0.001 \\
\hline
\end{tabular}

There was a highly significant statistical difference between the studied and control groups as regard one of the uterine artery Doppler indices: the pulsatility index (PI) in the late follicular phase ( $\rho$ : 0.001).Concerning the sub-endometrial blood flow, it was present in only $16.7 \%$ of the participants in group A versus 55\% in group B, while it was absent in $83.3 \%$ of the participants in group A versus $45 \%$ in group (B) (Table 4). Table 4: comparison of sub-endometrial blood flow between the studied and control groups

\begin{tabular}{|l|l|l|}
\hline & $\begin{array}{l}\text { Study } \\
\text { group } \\
(\mathbf{N}=30)\end{array}$ & $\begin{array}{l}\text { Control } \\
\text { group } \\
(\mathbf{N = 2 0})\end{array}$ \\
\hline $\begin{array}{l}\text { Subendometrial blood } \\
\text { flow Count Absent \% }\end{array}$ & $25(83.3 \%)$ & $9(45 \%)$ \\
\hline $\begin{array}{l}\text { Subendometrial blood } \\
\text { flow Count Present \% }\end{array}$ & $5(16.7 \%)$ & $11(55 \%)$ \\
\hline
\end{tabular}

The difference was statistically significant between the studied and control groups as regard of power Doppler image to the sub-endometrial blood flow in the late follicular phase ( $\rho: 0.018)$. Concerning the trilaminar pattern, it was present in $76.6 \%$ of the participants in group A versus $90 \%$ in group B, while it was absent in $23.3 \%$ of the participants in group A versus 10\% in group B (Table 5).

Table 5: comparison of trilaminar pattern between the studied and control groups

\begin{tabular}{|l|l|l|}
\hline & $\begin{array}{l}\text { Cases } \\
(\mathrm{N}=30)\end{array}$ & $\begin{array}{l}\text { Controls } \\
(\mathrm{N}=20)\end{array}$ \\
\hline
\end{tabular}




\begin{tabular}{|l|l|l|}
$\begin{array}{l}\text { Trilaminar NO Count } \\
\text { \%within group }\end{array}$ & $7(23.3 \%)$ & $2(10.0 \%)$ \\
\hline $\begin{array}{l}\text { Trilaminar Yes Count } \\
\text { \%within group }\end{array}$ & $\begin{array}{l}23 \\
(76.6 \%)\end{array}$ & $18(90.0 \%)$ \\
\hline \multicolumn{2}{|c|}{ The difference was statistically }
\end{tabular}
significant between the study and control groups as regard of trilaminar pattern in the late follicular phase ( $\rho$ : 0.006).

\section{DISCUSSION}

Ultrasound measurement of the endometrium is now an indispensable part of ovulation, induction, monitoring and assisted reproductive technologies. It also has a role in evaluation of unexplained infertility ${ }^{(3)}$. Before ultrasound, the condition of the endometrium could only be evaluated by progesterone challenge to induce withdrawal bleeding or by invasive procedures, biopsy, curettage, and hysteroscopy ${ }^{(15)}$. Angiogenesis plays a critical role in various female reproductive processes such as development of a dominant follicle, formation of a corpus luteum, growth of endometrium and implantation. Assessment of endometrial blood flow adds a physiological dimension to the anatomical ultrasound parameters ${ }^{\left({ }^{16}\right) . Z ̌ a ́ c ̌ k o v a ́ ~}$ et $\boldsymbol{a l} .{ }^{\left({ }^{(17)}\right.}$ conducted a study to evaluate whether endometrial or ovarian parameters as measured using 3D power Doppler ultrasound would predict the outcome in frozen embryo transfer (FET) cycles. Thirty women with no known gynecological pathology underwent FET were recruited. The FET was carried out in the natural menstrual cycle 3-4 days after the first positive LH test result. Three- dimensional (3D) ultrasonographic examination was performed on the day of the FET and repeated one week later. The demographic, clinical and embryological characters were similar between the pregnant $(15 / 30)$ and non pregnant groups $(15 / 30)$. There were no differences between the groups in endometrial/sub-endometrial thickness, volume, or vascularization index (VI). The endometrial triple-line pattern was more often present in the pregnant group on the day of the FET $(93.3 \%$ vs. $40.0 \%)$. At the second visit, the triple-line pattern was still more often present in those patients who had conceived (91.7\% vs. $42.9 \%)$. According to their results, measurement of power Doppler indices using 3D ultrasound on the day of the FET does not provide any additional information concerning the outcome of the cycle. The existence of the triple-line pattern on the day of the FET seems to be a prognostic sign of a prosperous outcome after FET and this is disagreed with our results as there were a significant statistical difference between fertile and infertile groups concerning endometrial thickness, triple-line pattern, and power Doppler of the subendometrial blood flow. Dechaud et al. ${ }^{(18)}$ conducted a study to determine when to perform transvaginal ultrasonography to evaluate uterine receptivity in IVF programmes. 124 patients with different causes of infertility, undergoing IVF or intra cytoplasmic sperm injection were studied. The ultrasonographic evaluation included endometrial thickness, endometrial pattern, uterine artery pulsatility index, and endometrial-subendometrial blood flow distribution pattern. All patients underwent ultrasonographic investigation on the days of human chorionic gonadotropin (HCG) administration, oocyte retrieval, and embryo transfer. The pregnancy and implantation rates per transfer were 33 and $19.8 \%$ respectively. In terms of single parameters, women with an endometrial- subendometrial blood flow and a multilayered endometrium were more likely to be pregnant than women without one or more of these signs. The most effective combination for evaluation of uterine receptivity was endometrial pattern and endometrial thickness. Sensitivity and specificity of this combination were around $81 \%$. The best sensitivity and specificity were obtained on the day of HCG administration: respectively 81.1 and $81.3 \%$.Their results agreed with our results as the fertile group had a good subendometrial blood flow in $56 \%$ versus $18 \%$ in the infertile group, and the fertile group had a trilaminar pattern in $88 \%$ versus $76.6 \%$ in the infertile group. Järvelä et $\boldsymbol{a l} .{ }^{\left({ }^{(19)}\right.}$ conduct a study to compare sonographic endometrial characteristics in in-vitro fertilization (IVF) cycles between women who conceive and those who do not, Thirty-five women with different causes of infertility undergoing IVF treatment participated in the study. Using threedimensional (3D) power Doppler ultrasound, they were assessed in endometrial patterns, volume and vascularization, after follicle stimulating hormone (FSH) stimulation but before human chorionic gonadotropin (HCG) administration and again on the day of oocyte retrieval. The pregnancy rate was $37 \%$ (13/35). After FSH stimulation, 29 of the 35 women had a triple-line endometrial pattern, compared with five out of 35 on the day of oocyte retrieval. In 
those who had a triple line pattern after FSH stimulation the pregnancy rate was $44.8 \%$ $(13 / 29)$ and it was $0 \%(0 / 6)$ in those with a homogeneous pattern $(\mathrm{P}=0.039)$. If a tripleline pattern was present on the day of oocyte retrieval the pregnancy rate was $80.0 \%$ (4/5), which agreed with our results, whereas if the pattern was homogeneous the pregnancy rate was $30.0 \%(9 / 30)(\mathrm{P}=0.032)$. There were no differences between those who conceived and those who did not in endometrial thickness, volume or vascularization on either day examined, which disagreed with our results. Endometrial volume decreased significantly after hCG injection in women who conceived, but not in those who did not conceive. In both groups endometrial and subendometrial vascularization decreased after hCG injection, while the endometrial thickness remained unchanged. They concluded that the existence of a homogeneous endometrial pattern after FSH stimulation seems to be a prognostic sign of an adverse outcome in IVF, while a tripleline pattern after FSH stimulation and a decrease in endometrial volume appear to be associated with conception.Abdel Kader $\boldsymbol{e t}$ al. ${ }^{(20)}$ concluded that, with endometrial thickness less than $8 \mathrm{~mm}$ and no-triple-line pattern on hCG day in IVF/ICSI cycles, there is a poor chance of achieving pregnancy. Average endometrial line thickness of 8-12 $\mathrm{mm}$ and triple line (good morphologic texture) are good prognostic values if good quality embryos are transferred, which agree with our results. On the other hand, Singh et al. (16) reported that, largest number of pregnancies occurred when the endometrial thickness was $8-10 \mathrm{~mm}$. They also postulated that no pregnancies reported when endometrial thickness is less than $5.8 \mathrm{~mm}$. Khan et al. ${ }^{(5)}$ reported that the pregnancy rate decreased as the endometrial thickness decreased. \& no pregnancy was reported when endometrial thickness was less than $7 \mathrm{~mm}$ which agree with our results. Okohue et $\boldsymbol{a l} .{ }^{(21)}$ concluded that endometrial thickness of at least $6.5 \mathrm{~mm}$ correlated with a higher rate of conception, while no pregnancy occurred in a similar study when the endometrial thickness was $<6 \mathrm{~mm}$. Casper ${ }^{(22)}$ speculated that thinner endometrium resulting in implantation failure may be related to oxygen tension. When the thickness measured by ultrasound is $<7 \mathrm{~mm}$, the functional layer is thin or absent, and the implanting embryo would be much closer to the spiral arteries and the higher vascularity and oxygen concentrations of the basal endometrium. The high oxygen concentrations near the basal layer could be detrimental compared with the usual low oxygen tension of the surface endometrium ElZenneni et al. ${ }^{(23)}$ stated that the triple layer endometrial pattern was the most suitable for conception which agree with us. Also Singh $\boldsymbol{e t}$ al. ${ }^{(16)}$ reported that the triple layer endometrium was a good prognostic factor for occurrence of pregnancy. Zhao et al. ${ }^{(24)}$ concluded that endometrial thickness and pattern independently affect pregnant outcomes which disagree with our results. Combined endometrial thickness and pattern could not predict the outcome of IVF-ET when endometrial thickness was $<7$ or $>14 \mathrm{~mm}$, while a triple-line pattern with a moderate endometrial thickness appeared to be associated with a good clinical outcome. Nygren et al. ${ }^{(25)}$ had suggested positive correlation of the endometrial and subendometrial blood flows to the outcome of IVF which supports our results, while Aghahoseini et al. ${ }^{(26)}$ had suggested negative correlation. Khan et al. ${ }^{(5)}$ concluded that, the pregnancy rate was significantly higher in patients with multifocal vascularity in zone 3. Similar finding was reported by Chien et al. (6). A study was done by Apollo-Assisted Reproduction Unit revealed the significant correlation between pregnancy rate and endometrial vascularity, absent endometrial vascularization was associated with no conception which agree with our results. Schild et al. ${ }^{(27)}$ evaluated 96 patients undergoing IVF program by 3D-PDA. Ultrasound examination was performed on the first day of ovarian stimulation after pituitary down regulation. Pregnancy rate was $20 \%$. Only subendometrial vascularization was assessed, but the authors provided no definition of "subendometrial region". They found that all 3D-PDA indices were significantly lower in conception with non-conception cycles. However, a great overlapping existed.

Kupesic ${ }^{\left({ }^{8}\right)}$ assessed 89 women by 3DPDA the day of embryo transfer. Subendometrial region was defined as $5 \mathrm{~mm}$ outer from endometrial defined borders. Pregnancy rate was $31.5 \%$. Subendometrial FI (flow index) was significantly higher in the pregnancy group which gets similar with our results. Wu et al. ${ }^{(28)}$ evaluated prospectively 54 patients undergoing IVF ET. 3D-PDA evaluation was performed on the day of hCG administration. Subendometrial region was 
defined as $5 \mathrm{~mm}$ surrounding the endometrial borders. Pregnancy rate was $50 \%$. They did not find differences on subendometrial VI and FI between conception and non-conception cycles. However, subendometrial VFI was significantly higher in those patients who become pregnant, which agree with our results. Alcazar ${ }^{(29)}$ addressed some technical aspects of 3D US and reviewed critically its current status in evaluating endometrial function with special focus in its role in predicting pregnancy in assisted reproductive techniques. The author found that in spontaneous cycles endometrial volume grows during follicular phase remaining constant through the luteal phase. Endometrial vascularization increases during follicular phase peaking 2-3 days before ovulation, decreasing thereafter and increasing again during mid and late luteal phase. He also found that data from studies analyzing the role of 3D US for predicting IVF outcome are controversial. An explanation for these controversial findings might be different design of reported studies, specially the timing of ultrasound evaluation. Chien et al. ${ }^{\left({ }^{(6)}\right.}$ conduct a study to investigate changes in utero-ovarian blood flow during the peri-implantation period and their significance in successful embryo implantation. A prospective longitudinal study was conducted in 317 women undergoing in vitro fertilization-embryo transfer (IVF-ET) treatment. Measurement of endometrial thickness and color flow imaging with pulsed waveform analysis of uterine and ovarian arteries were performed before ET and 5-6 days after ET. Pregnancy was achieved in 91 of the study patients. There was no difference in mean endometrial thickness between the two groups before ET, which disagree with our results, while a thicker endometrium was found in women who had conceived compared with those who had not 5-6 days after ET $(\mathrm{P}=0.02)$. Mean uterine arterial resistance index (RI) and pulsatility index (PI) values were significantly lower in the pregnant than in the non-pregnant group before ET $(\mathrm{P}=0.04$ and $\mathrm{P}=0.003$, respectively), but no significant differences were found between the two groups 5-6 days after ET. Different utero-ovarian blood flow changes during the peri-implantation period occur in conception and non-conception cycles in women following IVF. Doppler assessment of uterine arterial resistance can help to determine a time interval within the menstrual cycle that is of optimal endometrial status for embryo implantation in assisted conception programs. Delay in achieving adequate uterine perfusion during the temporal window of embryo implantation may have an impact on endometrial receptivity. Carbillon et al. (30) supported our results as they reached a conclusion of their study that the assessment of uterine artery flow by Doppler is easy and reproducible, and high impendence at the end of the follicular phase is a good indicator of poor endometrial receptivity.

\section{CONCLUSION}

Ultrasound as well as Doppler studies of endometrial and subendometrial vascularization has a role in predicting endometrial receptivity. Uterine artery Doppler study and endometrial thickness measurement have a role in detection of uterine receptivity. Ultrasonographic endometrial makers of receptivity can be used to help in detecting patients with failed implantation as a cause of unexplained infertility or failed assisted conception.The study of ultrasonographic endometrial makers of receptivity still needs a vast randomized controlled trials including large number of participants and meta-analysis to evaluate the possibility of setting a scoring system that can be useful in assisted reproductive programs as a predictor of uterine receptivity.

\section{REFERENCES}

1. Practice Committee of the American Society for Reproductive Medicine (ASRM) (2006): Effectiveness and treatment for unexplained infertility. Fertil. Steril., 86: 111-115.

2. El-Mazny A, Abou-Salem $\mathbf{N}$ and Elshenoufy H (2013): Doppler study of uterine hemodynamics in women with unexplained infertility. Eur $\mathbf{J}$ Obstet Gynecol Reprod Biol., 171: 84-87.

3. Kasius A, Smit J, Torrance $\mathrm{H}$ et al. (2014): Endometrial thickness and pregnancy rates after IVF: A systematic review and meta-analysis. Hum. Reprod. Update, 20: 530-541.

4. Martins WD, Ferriani RA, Nastri CO et al. (2008): Measurement of endometrial volume increase during the first week after embryo transfer by three-dimensional ultrasound to detect pregnancy: a preliminary study. Fertil. Steril., 90(3): 883-885.

5. Khan MS, Shaikh A and Ratnani $R$ (2016): Ultrasonography and Doppler 
study to predict uterine receptivity in infertile patients undergoing embryo transfer. J. Obstet .Gynaecol. India, 66(1): 377-382.

6. Chien L W, Lee WS, Au HK et al. (2004): Assessment of changes in uteroovarian arterial impedance during the periimplantation period by Doppler sonography in women undergoing assisted reproduction. Ultrasound Obstet. Gyneco1., 23 (5): 496-500.

7. Knowlton NS, Craig LB, Zavy MT et al. (2014): Validation of the power model of ovarian nongrowing follicle depletion associated with aging in women. Fertil. Steril., 101: 851-856.

8. Kupesic S (2004): Three-dimensional ultrasonographic uterine vascularization and embryo implantation. J. Gynecol. Obstet. Biol. Reprod. (Paris), 33(2): 1820.

9. Lindhard A, Bentin-Ley $U$ and Ravn $V$ (2002): Biochemical evaluation of endometrial function at the time of implantation. Fertil. Steril., 78: 221-233.

10. Fanchin $R$ (2001): Assessing uterine receptivity in: ultrasonographic glances at the new millennium. Ann. Acad. Sci., 943:185-187.

11. Mahajan N (2015): Endometrial receptivity array: clinical application. J. Hum. Reprod. Sci., 8(3): 121-129.

12. James JL, Carter AM and Chamley LW (2012): Human placentation from nidation to 5 weeks of gestation. Part I: What do we know about formative placental development following implantation? Placenta, 33: 327-334.

13. Thomson AJM, Holland $\mathbf{N}$ and Kingsland CR (2002): Factors affecting embryo implantation in fertilization in vitro. In: Book of Obstetric and Gynecology. Sturadeed Olahk Purdid.USA. pp: 368-373.

14. Terakawa J, Watanabe $T$, Obara $R$ et al. (2012): The complete control of murine pregnancy from embryo implantation to parturition. Reproduction, 143(3): 411-416.

15. Dickey PR (2010): Ultrasonography of the Endometrium for Infertility. Cambridge University Press, PP:137-142.

16. Singh $\mathrm{N}$, Bahadur $\mathrm{A}$, Mittal $\mathrm{S}$ et al. (2011): Predictive value of endometrial thickness, pattern and sub-endometrial blood flows on the day of hCG by $2 \mathrm{D}$ doppler in in-vitro fertilization cycles: A prospective clinical study from a tertiary care unit. J Hum Reprod Sci., 4(1): 29-33.

17. Žáčková T, Järvelä YI, Tapanainen SJ et al. (2009): Assessment of endometrial and ovarian characteristics using three dimensional power Doppler ultrasound to predict response in frozen embryo transfer cycles. Reproductive Biology and Endocrinology, 7: 151-160.

18. Dechaud H, Bessueille E, Bousquet PJ et al. (2008): Optimal timing of ultrasonographic and Doppler evaluation of uterine receptivity to Implantation. Reprod. Biomed. Online, 16(3): 368-375.

19. Järvelä IY, Sladkevicius $P$, Kelly $S$ et al. (2005): Evaluation of endometrial receptivity during in-vitro fertilization using threedimensional power Doppler ultrasound. Ultrasound in Obstetrics and Gynecology, 26: 765-769.

20. Abdel Kader M, Abdelmeged A, Mahran A et al. (2016): The usefulness of endometrial thickness, morphology and vasculature by 2D Doppler ultrasound in prediction of pregnancy in IVF/ICSI cycles. The Egyptian Journal of Radiology and Nuclear Medicine, 47(1): 341-346.

21. Okohue JE, Onuh SO, Ebeigbe $P$ et al. (2009): The effect of endometrial thickness on in vitro fertilization (IVF)embryotransfer/ intracytoplasmic sperm injection (ICSI) outcome. Afr. J. Reprod. Health, 1: 113-121.

22. Casper RF (2011): It's time to pay attention to the endometrium. Fertil. Steril., 96(3): 519-521.

23. El-Zenneni $\mathbf{H}$, Moustafa $\mathbf{R}$, AbdelHafeez $M$ et al. (2014): Assessment of uterine, subendometrial blood flows and endometrial gland vascular endothelial growth factor (EG-VEGF) in women with unexplained infertility. Middle East Fertil. Soc. J., 20(2): 119-126.

24. Zhao J, Zhang $Q$ and Li Y (2012): The effect of endometrial thickness and pattern measured by ultrasonography on pregnancy outcomes during IVF-ET cycles. Reprod. Biol. Endocrinol., 28(10): 100-106.

25. Nygren KG, Sullivan E, ZegersHochschild F et al. (2011): International committee for monitoring assisted reproductive technology (ICMART) 
world report: assisted reproductive technology. Fertil. Steril., 95: 2209-2222

26. Aghahoseini M, Tuba K, Marsousi V et al. (2008): Assessment of endometrialsubendometrial blood flow detected by color Doppler sonography and uterine receptivity in infertile women. Acta Med. Iran, 46: 461-466.
27. Schild RL, Holthaus S, d'Alquen J et al. (2000): Quantitative assessment of subendometrial blood flow by threedimensional-ultrasound is an important predictive factor of implantation in an invitro fertilization programme. Hum. Reprod., 15: 89-94.

28. Wu HM, Chiang CH, Huang HY et al. (2003): Detection of the subendometrial vascularization flow index by threedimensional ultrasound may be useful for predicting the pregnancy rate for patients undergoing in vitro fertilization - embryo transfer. Fertil. Steri1., 79(3): 507-511.

29. Alcazar JL (2006): Three-dimensional ultrasound assessment of endometrial receptivity: a review. Reprod. Biol. Endocrinol., 4: 56-62.

30. Carbillon $\mathrm{L}$, Perrot $\mathrm{N}$, Uzan $\mathrm{M}$ et al. (2001): Doppler ultrasonography and implantation: a critical review. Fetal Diagn. Ther., 6 (6): 327-332. 